\title{
Exercise training and dietary restriction affect PINK1/Parkin and Bnip3/Nix-mediated cardiac mitophagy in mice
}

\author{
Yongcai Zhao ${ }^{1}$, Qiaodi Zhu' ${ }^{1}$, Wenjuan Song ${ }^{1}$ and Binghong $\mathrm{Gao}^{2}$ \\ ${ }^{1}$ Graduate Department of Shanghai University of Sport, Shanghai, PR China \\ ${ }^{2}$ School of Physical Education and Sport Training, Shanghai University of Sport, Shanghai, PR China
}

\begin{abstract}
The aim of this study was to investigate the effects of exercise training and dietary restriction on cardiac PINK1/Parkin and Bnip3/Nix pathways involved in mitophagy. C57BL/6 mice were assigned to control (C), exercise training (T), dietary restriction (D) and exercise training + dietary restriction (TD) groups. T group undertook 10 weeks of swimming training. D group was subjected to $40 \%$ food reduction. TD group undertook the combination of exercise training and food reduction. Suspectable mitophagy autophagosomes can be observed in T and D groups and more autophagosomes appeared in TD group. In T group, both PINK1 mRNA and protein levels increased significantly $(p<0.01)$ but Bnip3 and Nix expressions decreased significantly $(p<0.05)$. Dietary restriction resulted in elevation of Drp1 $(p<0.01)$ and reduction and Nix $(p<0.05)$ in $\mathrm{D}$ group. The load in TD group resulted in serious mitochondrial abnormalities and myofibrillar damages accompanied by increases in PINK1 and Drp1 levels $(p<0.01)$ and decreases in Bnip3 and Nix levels $(p<0.05)$. The increase in PINK1 expression (exercise protocol) or Drp1 expression (diet protocol) contributes to cardiac activation of mitophagy, whereas Bnip3 and Nix are not implicated in this activation.
\end{abstract}

Key words: Swimming - Mitochondrial autophagy - Cardiomyocyte - Calorie restriction

\section{Introduction}

Autophagy is a process by which dysfunctional cytosolic constituents are segregated by double membrane vesicles called "autophagosomes", which then fuse with lysosomes to degrade the cargo materials for future use (Choi et al. 2013). Autophagy degrades damaged materials massively that can be accelerated for the requirement of materials especially in nutrients deprivation. Autophagy was initially considered as a non-selective process, but recently, several forms of organelle-specific autophagy have been identified, implying that cell can selectively remove specific organelle (Quinsay et al. 2010). Of these, autophagic removal of mitochondria, termed "mitophagy", has been intensively studied. Mitophagy refers to the mechanism by which cell degrades and recycles damaged mitochondria in lysosome-dependent

Correspondence to: Binghong Gao, School of Physical Education and Sport Training, Shanghai University of Sport, Changhai road 399, Yangpu District, Shanghai 200438, PR China

E-mail: gaobinghong@126.com engulfment manner, maintaining cellular homeostasis (Tolkovsky 2009).

In human heart, mitochondria account for approximately $30 \%$ cardiomyocyte volume and produce nearly $6 \mathrm{~kg}$ adenosine triphosphate (ATP) every day to support physiological functions (Hall et al. 2014). During this process, as a byproduct, reactive oxygen species (ROS) leak from electron transport chain and exert positive function in normal condition. But excessive ROS generated from pathophysiological stresses such as senescence and ischemia reperfusion (IR) attack mitochondrial DNA (mtDNA), lipids and proteins, resulting in mitochondrial dysfunction (Youle and van der Bliek 2012). Because ROS-induced accumulation of impaired mitochondria readily facilitates apoptotic and necrotic processes, it is essential for cardiac mitochondria to maintain healthy condition due to the energetical sensitiveness and high energy demand of cardiac function (Dorn 2016). Genetic manipulation inhibiting mitophagy enhances cardiomyocyte death and myocardial injury in response to IR (Ikeda et al. 2015), suggesting that cardiac mitophagy plays an essential role in removing the damaged mitochondria. Although mitophagy 
shares most of molecular mechanism with non-selective autophagy, mitophagy has unique signaling pathways, in which the most explored molecular machinery is PINK1/Parkin pathway (Youle and Narendra 2011). When mitochondria deteriorate upon stress, PTEN-induced putative protein kinase 1 (PINK1) accumulates on outer mitochondrial membrane (OMM) and phosphorylates target proteins, then recruiting Parkin (a cytosolic E3 ubiquitin ligase) to localize on OMM and initiating mitochondrial elimination in cardiomyocytes (Chen and Dorn 2013). Additionally, BCL2 and adenovirus E1B 19-kDa-interacting protein 3 (BNIP3) and BNIP3-like (Bnip3L or Nix), as mitophagy receptors, are mitochondrialocalized proteins and interact with microtubule-associated protein 1 light chain 3 (LC3) through their LC3-interacting region (LIR), thus promoting mitochondrial clearance in cardiomyocytes (Dorn 2010).

During past years, extensive investigations involving genetic manipulation on mitophagy have been accomplished in cultured cells and vivo models. A review from Shires et al. indicates that PINK/Parkin- and Bnip3/Nix-mediated mitophagy are crucial in maintaining cardiac homeostasis (Shires and Gustafsson 2015). Moreover, cardiac mitochondria also undergo fusion and fission to coordinate with mitophagy, thereby efficiently removing impaired mitochondria. Mitochondrial fusion protein Mfn2, as a Parkin receptor, mediates recruitment of Parkin, and fission protein dynamin-related protein 1 (Drp1) promotes mitochondrial asymmetrical fission for elimination of the damaged mitochondria (Shirihai et al. 2015). Thus, mitochondrial remodeling of structure cooperates with mitophagy constantly to help cardiomyocyte stay alive.

Currently, it is well established that nutrients deficiency can enhance autophagy in various tissues (Szafranski and Mekhail 2014). Exercise also increases autophagy in skeletal muscle which was reviewed by Rocchi et al. (Rocchi and He 2017), but exercise-induced alterations in mitophagy in muscle have less been documented, particularly in cardiac tissues. It is unknown whether exercise training and dietary restriction can upregulate cardiac mitophagy, producing beneficial effects in cardiomyocytes. Therefore, we focused on PINK/Parkin and Bnip3/Nix pathways and aimed to examine the effects of exercise and dietary restriction on cardiac mitophagy, potentially providing insight into the strategies in maintenance of cardiac healthy.

\section{Materials and Methods}

\section{Animal treatments and exercise protocols}

Male C57BL/6 mice (eight weeks) were housed in a 12:12-h light-dark cycle and given water ad libitum and standard mouse food. They were randomly assigned into control (C), exercise training (T), dietary restriction (D) and exercise training + dietary restriction (TD) groups, $n=8$ /group. All experimental procedures were approved by the Research Commission on Ethics of Shanghai University of Sport and conducted in accordance with the Guide for the Care and Use of Laboratory Animals (NIH Publication No. 85-23). Animals (T group) performed 10 weeks of increased load swimming training (5 days/week). Duration time in first week was 20 min every day and then increased 10 min per week. Last 3 weeks duration was maintained at $90 \mathrm{~min} / \mathrm{per}$ day. Animals (D group) were fed food equivalent to $60 \%$ intake of control group as conducted in previous study (Watanabe et al. 2014). Average food intake a day was calculated every week in $\mathrm{C}$ group and mice in D group received $60 \%$ the intake in the next week. Duration of dietary intervention was identical to T group. Main compositions of Specific Pathogen Free (SPF) food included 9\% water, 23\% protein, $6 \%$ fat and $4 \%$ crude fibre. In the first week, a mouse consumed average $2.5 \mathrm{~g}$ food in $\mathrm{C}$ group. The average consumed food fluctuated from 3.0-4.5 g from second week to end of experiment. Animals (TD group) were subjected to the combination intervention of training and dietary restriction just as described in $\mathrm{T}$ and $\mathrm{D}$ groups. Heart samples were harvested $24 \mathrm{~h}$ post final exercise. Approximate $1 \mathrm{~mm}^{3}$ left ventricle tissue was retained in glutaraldehyde fixed solution for electron microscope observation. Another ventricle tissue (left and right ventricles) was immediately frozen in liquid nitrogen and stored at $-80^{\circ} \mathrm{C}$ for gene and protein analysis.

\section{Gene expression analysis}

Quantitative real-time PCR was used to evaluate mRNA expression. Total RNA was isolated by Trizol reagent (Invitrogen) and OD260/OD280 > 1.8. cDNA was synthesized using SuperScript III FirstStrand synthesis kit (Invitrogen) according to the manufacturer's instructions. The primers were designed on the information of sequences available in GenBank and shown in Table 1.

PCR was performed using ABI PRISM 7500 Sequence Detection System (Applied Biosystems) according to the manufacturer's protocol. GAPDH was used as housekeeping gene. PCR was performed using cDNA and specific primers for 40 cycles. The $20 \mu \mathrm{l}$ PCR reaction liquid included $5 \mu \mathrm{l}$ cDNA, forward primer $0.5 \mu \mathrm{l}$ and reverse primer $0.5 \mu \mathrm{l}$, $10 \mu \mathrm{l}(2 \times)$ SYBR Green qPCR SuperMix (Invitrogen) and $4 \mu \mathrm{l}$ nuclease-free water. The reactions were incubated in 96-well-plate at $50^{\circ} \mathrm{C}$ for $2 \mathrm{~min}, 95^{\circ} \mathrm{C}$ for $2 \mathrm{~min}$, and then, followed by 40 cycles of $95^{\circ} \mathrm{C}$ for $15 \mathrm{~s}$ and $60^{\circ}$ for $32 \mathrm{~s}$. mRNA expressions was normalized by GAPDH expression. Threshold cycle $(\mathrm{Ct})$ was set within the exponential phase of the PCR. Ct values of genes were normalized by subtracting $\mathrm{GAPDH} C \mathrm{Ct}$ value, providing the $\Delta \mathrm{Ct}$ value. Relative gene expression $=2^{-(\Delta \text { Ctsample- } \Delta \text { Ctcontrol })}$. 
Table 1. Sequences of primers for the genes regulating mitophagy

\begin{tabular}{lcc}
\hline \multirow{2}{*}{ Genes } & \multicolumn{2}{c}{ Sequences (5'-3') } \\
\cline { 2 - 3 } & \multicolumn{2}{c}{ Forward } \\
\hline Drp1 & AAATTGGGCTCCAAGACTCA & CTGTTGGCGTGGAACTAAAG \\
Parkin & GTTCGTCTCATCATGGATTC & TTAATTCGCAGCTTGTAGTC \\
Pink1 & CACCTGCAAACAAGCAAC & CACTCATCCGGTTTGGAAT \\
Bnip3 & GGCAGAATTAAGACATCAGG & TTGCCTCAGTGATAGGTTAG \\
Nix & GCATGAATCTGGACGAAGTA & AGCTCTGTTGGTATCTTGTG \\
GAPDH & GAGCACAGCATCACTCTGAA & AGCCTTTGTGAGAGAGGAGT \\
\hline
\end{tabular}

\section{Western blotting analysis}

Cardiac samples in RIPA buffer (Beyotime Biotech) were placed on ice for $20 \mathrm{~min}$ and centrifuged at $12,000 \times \mathrm{g}$ for $20 \mathrm{~min}$. Proteins were separated by SDS-PAGE and then transferred to PVDF membranes. Membranes were incubated overnight at $4^{\circ} \mathrm{C}$ with primary antibody. GAPDH (Santa Cruz Biotechnology), Drp1 (Cell Signaling Technology), PINK1 (Proteintech Group), Parkin, Mfn2, Bnip3 and Nix antibodies (Boster Biological Technology) were used. After washing, membranes were incubated with horseradish peroxidase-conjugated secondary antibody and visualized with enhanced chemiluminescence. Quantification was performed with Image J software. GAPDH levels were used to normalize the results.

\section{Transmission electron microscopy}

Cardiac tissues were collected and fixed in $2.5 \%$ glutaraldehyde for at least $24 \mathrm{~h}$. After washed with $0.1 \mathrm{M}$ sodium cacodylate buffer, tissues were immersed in $2 \%$ osmium tetroxide and $1 \%$ aqueous uranyl acetate, each immersion for $1 \mathrm{~h}$. Tissues were then dehydrated with ethanol solutions ( $50 \%, 70 \%, 90 \%$ and $100 \%$, each for $15 \mathrm{~min}$ ), tissues were transferred to propylene oxide, incubated in 1:1 propylene oxide and Embed 812 for $1 \mathrm{~h}$. Sections $(70 \mathrm{~nm})$ were cut by Leica ultramicrotome (UC-6). Electron microscope (Hitachi-7650, Japan) was used for ultrastructural examination of organelles. Gatan CCD system was applied to get image.

\section{Statistical analysis}

Statistical analyses were conducted using SPSS-Statistics package Version 19.0. The values are presented as means \pm SD. Two-way ANOVA was performed to evaluate the data, followed by Tukey's test for post hoc test. Statistical significance was set at $p<0.05$.

\section{Results}

Comparisons of body and heart weight in different groups after interventions

As shown in Table 2, there is no difference in baseline body weight among 4 groups $(p>0.05)$. After intervention, the changes in body weight are significant different among 4

Table 2. Comparisons of body and heart weight after interventions

\begin{tabular}{|c|c|c|c|c|}
\hline \multirow{2}{*}{ Parameter } & \multicolumn{4}{|c|}{ Group } \\
\hline & $\mathrm{C}$ & $\mathrm{T}$ & $\mathrm{D}$ & $\mathrm{TD}$ \\
\hline Pre-body weight (g) & $21.65 \pm 0.46$ & $22.02 \pm 1.04$ & $21.58 \pm 0.95$ & $21.64 \pm 1.15$ \\
\hline Post-body weight (g) & $30.18 \pm 1.28$ & $26.88 \pm 1.56^{* *}$ & $22.98 \pm 0.79^{* *}$ & $22.81 \pm 1.52^{\star *}$ \\
\hline Weight change (g) & $8.53 \pm 1.59$ & $4.86 \pm 1.70^{* *}$ & $1.40 \pm 1.11^{\star *}$ & $1.18 \pm 1.42^{* *}$ \\
\hline Heart weight (mg) & $144.80 \pm 5.12$ & $145.13 \pm 7.61$ & $129.77 \pm 7.06^{* *}$ & $122.12 \pm 8.92^{\star *}$ \\
\hline Heart/body ratio (mg/g) & $4.81 \pm 0.32$ & $5.42 \pm 0.29^{* *}$ & $5.65 \pm 0.41^{\star *}$ & $5.35 \pm 0.52^{\star *}$ \\
\hline
\end{tabular}

${ }^{* *} p<0.01$ vs. C group, $n=8$ /group. C, control group; T, exercise training group; $\mathrm{D}$, dietary restriction group; TD exercise training + dietary restriction group; Pre-body weight, body weight pre-intervention; Post-body weight, body weight post-intervention. 
groups. Post-hoc analysis showed that increases of body weight in $\mathrm{T}, \mathrm{D}$ and $\mathrm{TD}$ groups were lower than that in C group (all $p<0.01$ ). Heart weights in $\mathrm{D}$ and TD groups also were lower than that in C group $(\mathrm{p}<0.01)$. Heart and body weight ratios in T, D and TD groups were higher than that in C group $(p<0.01)$. The higher heart and body weight ratios in T, D and TD groups were mainly attributed to the slow increases of body weight in these groups compared with $\mathrm{C}$ group.

Subcellular structures change obviously in response to exercise and dietary restriction

Transmission electron microscopy was used to observe ultrastructural features upon exercise and dietary stresses. Myofibrils $\mathrm{Z}$ lines and intercalated disc structures in $\mathrm{C}$ and $\mathrm{T}$ groups were clear and intact, and tightly-packed mitochondria between myofibrils exhibited normal condition, particularly in $\mathrm{T}$ group. $\mathrm{Z}$ lines in $\mathrm{D}$ group exhibited slight curve but mitochondrial morphology did not alter. Surprisingly, TD group lacked sufficient intact $Z$ lines and abnormal mitochondria increased obviously. Mitochondria showed swelling and lost integrities of mitochondrial crista, suggesting that pathological injuries occurred in TD group
(Fig. 1). There were few autophagosomes in $\mathrm{C}$ group but the autophagosomes enclosing suspectable mitochondria could be found in T, and D groups, particularly more autophagosomes appeared in TD group (Fig. 2). Taken together, exercise training or dietary restriction resulted in increased mitophagy and the combined invention led to higher mitophagy level.

The change of PINK1/Parkin pathway in response to exercise training and dietary restriction

As illustrated in Figure 3, PINK1 expressions in T and TD groups were upregulated by $146 \%(p<0.01)$ and $116 \%(p<$ 0.01 ), respectively, compared with C group (Fig. 3A). PINK1 mRNA expressions also exhibited the same changes $(22 \%$ and $36 \%$ increase in T and TD groups, respectively, $p<0.01$ ) (Fig. 3C). Parkin protein expressions exhibited decreased trends in T, D and TD groups $(p>0.05)$ (Fig. 3B) but the Parkin mRNA expression was reduced significantly by $27 \%$ in TD group compared with $\mathrm{C}$ group $(p<0.05)$ (Fig. 3D). The abovementioned findings indicated that, in PINK1/ Parkin pathway, PINK1 factor is more sensitive than Parkin following exercise training and the combination intervention in TD group.
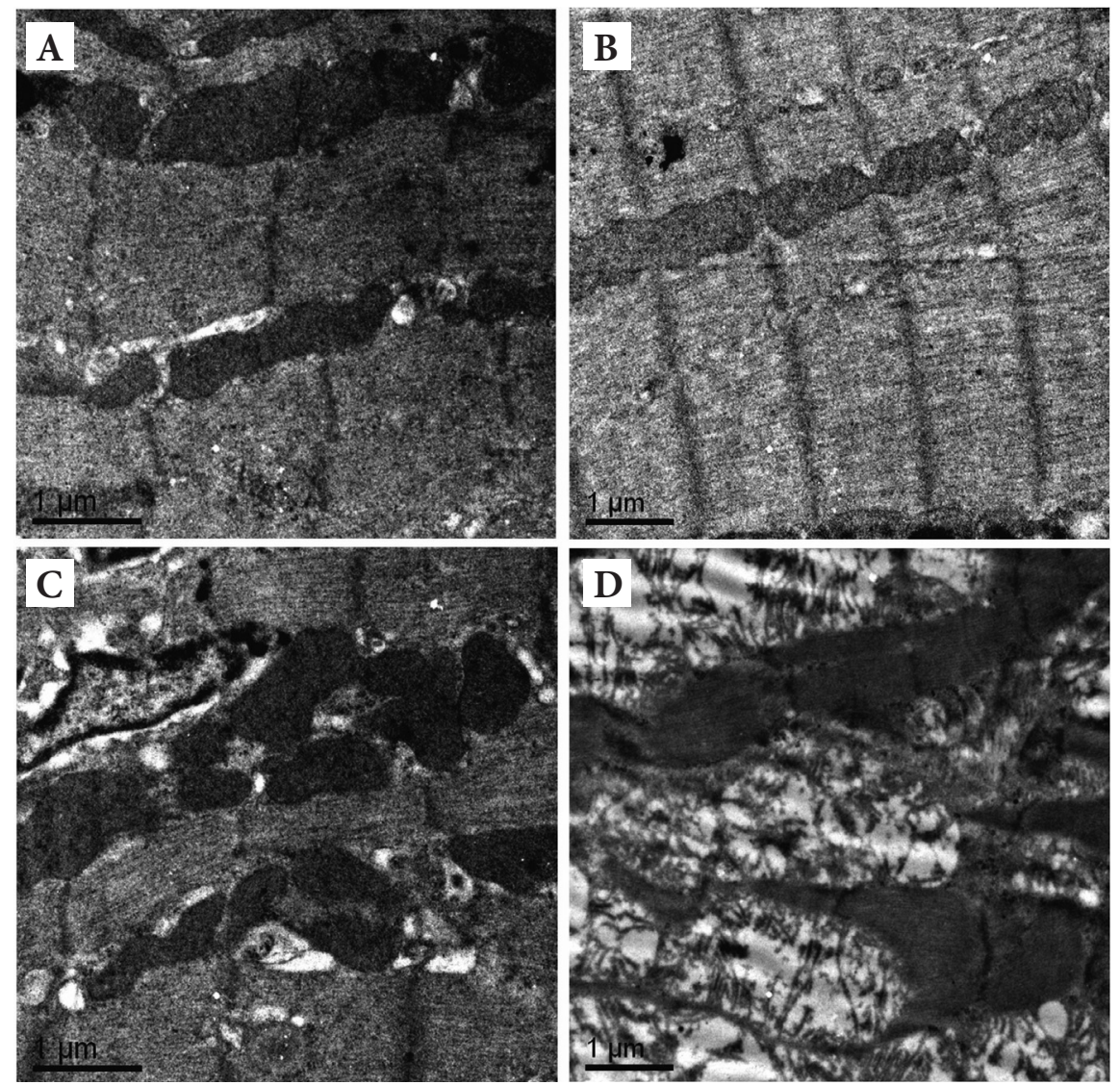

Figure 1. Transmission electron microscopy shows the mitochondria and myofibrils of control group (A), exercise training group $(\mathbf{B})$, dietary restriction group $(\mathbf{C})$ and exercise training + dietary restriction group (D). 

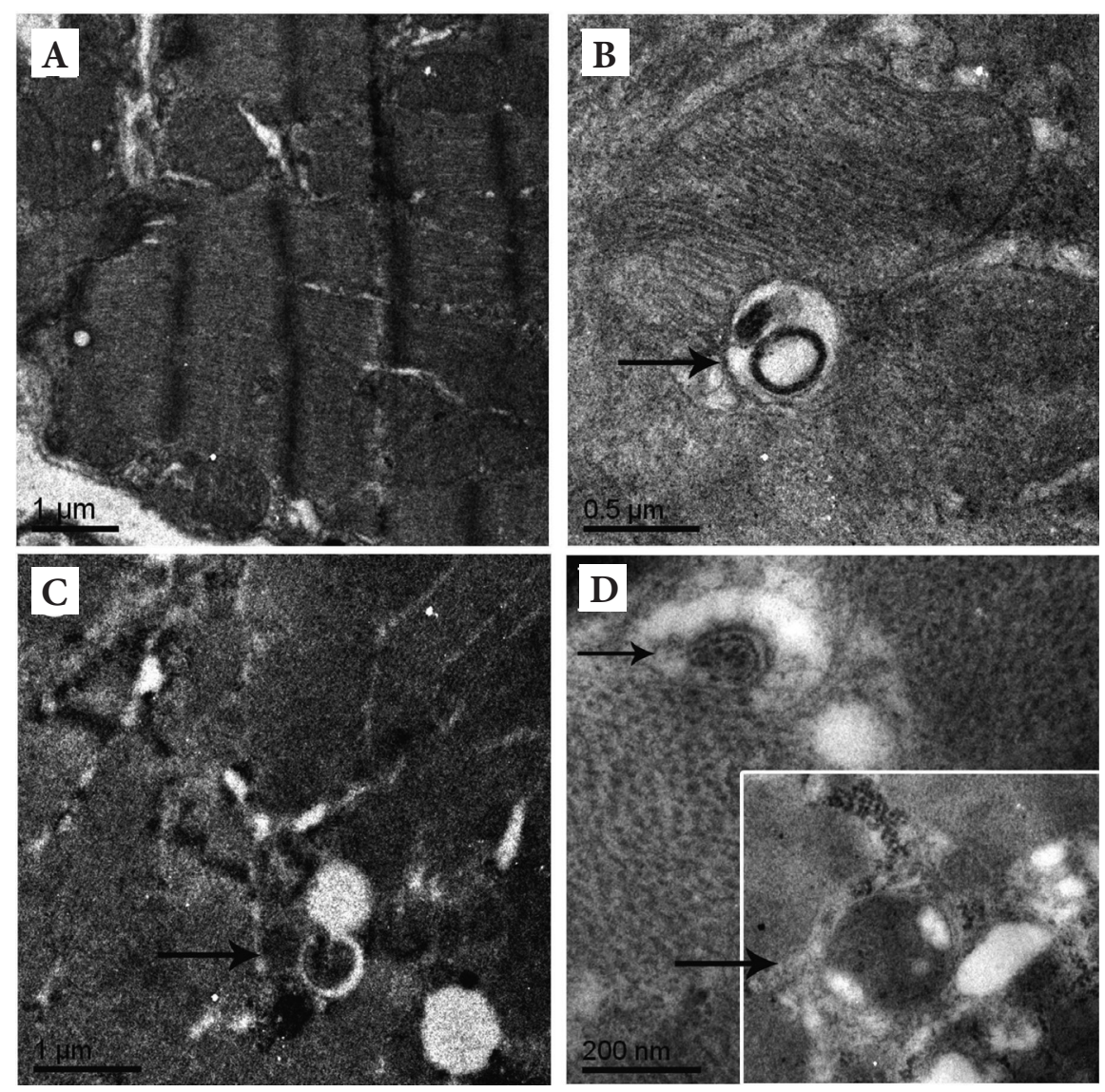

Figure 2. Transmission electron microscopy shows the status of mitochondrial autophagy. Representative images of control group (A), exercise training group (B), dietary restriction group (C) and exercise training + dietary restriction group (D). Black arrows point to the suspectable autophagosomes enclosing mitochondria.

The change of mitophagy receptor pathway in response to exercise training and dietary restriction

We tested whether Bnip3 and Nix are involved in mitophagy induced by exercise and dietary interventions. Fig. 4A illustrated the differences in Bnip3 protein levels among four groups. Compared with C group, Bnip3 levels were reduced by $42 \%$ and $45 \%$ in T and TD groups, respectively $(p<0.05)$ and the reduction in D group had not statistical significance $(p>$ 0.5). Change of Bnip3 mRNA expression had decreased trends (15\% and $16 \%$ reductions in T and TD groups, respectively $(p$ $>0.5$ ) (Fig. 4C). Nix protein expression decreased significantly in three groups $(37 \%, 36 \%$ and $44 \%$ reductions in $\mathrm{T}, \mathrm{D}$ and TD groups, respectively, $p<0.05)$ compared with $\mathrm{C}$ group (Fig. 4B). Nix mRNA expression exhibited the same change as Nix protein and the levels were reduced by $36 \%, 34 \%$ and $40 \%$ in T, D and TD groups respectively $(p<0.05)$ (Fig. 4D).

The change of mitochondrial dynamics protein in response to exercise training and dietary restriction

We investigated whether the mitochondrial dynamics proteins involved in mitophagy can be changed by exercise training and dietary restriction. Compared with $\mathrm{C}$ group, Mfn2 appeared to be resistant to all intervention protocols in the current study because neither protein nor mRNA expression was affected significantly $(p>0.05)$ (Fig. 5A, 5C). In contrast, Drp1 levels were upregulated by $27 \%, 68 \%$ and $85 \%$ in T, D and TD groups, respectively. Moreover, increases in $\mathrm{D}$ and TD groups had the statistical significance $(p<0.05)$ (Fig. 5B). Drp1 mRNA levels exhibited increased trend in T group and decreased trend in D group $(p>0.05)$, and the increase in TD group reached the statistical significance $(45 \%$ increase, $p<0.05$ ) (Fig. 5D).

\section{Discussion}

Heart is a highly energetically-demanding organ and cardiomyocytes synthesized ATP mainly through mitochondrial oxidative phosphorylation accompanied by ROS production. Prolonged high levels of ROS induced by harmful stresses induce oxidative stress, which produces more ROS in a vicious circle and easily leads to cardiomyocytes loss (Baines 2010). Terminally differentiated cells, such as cardiomyocytes, are more highly dependent on organelles quality control than 

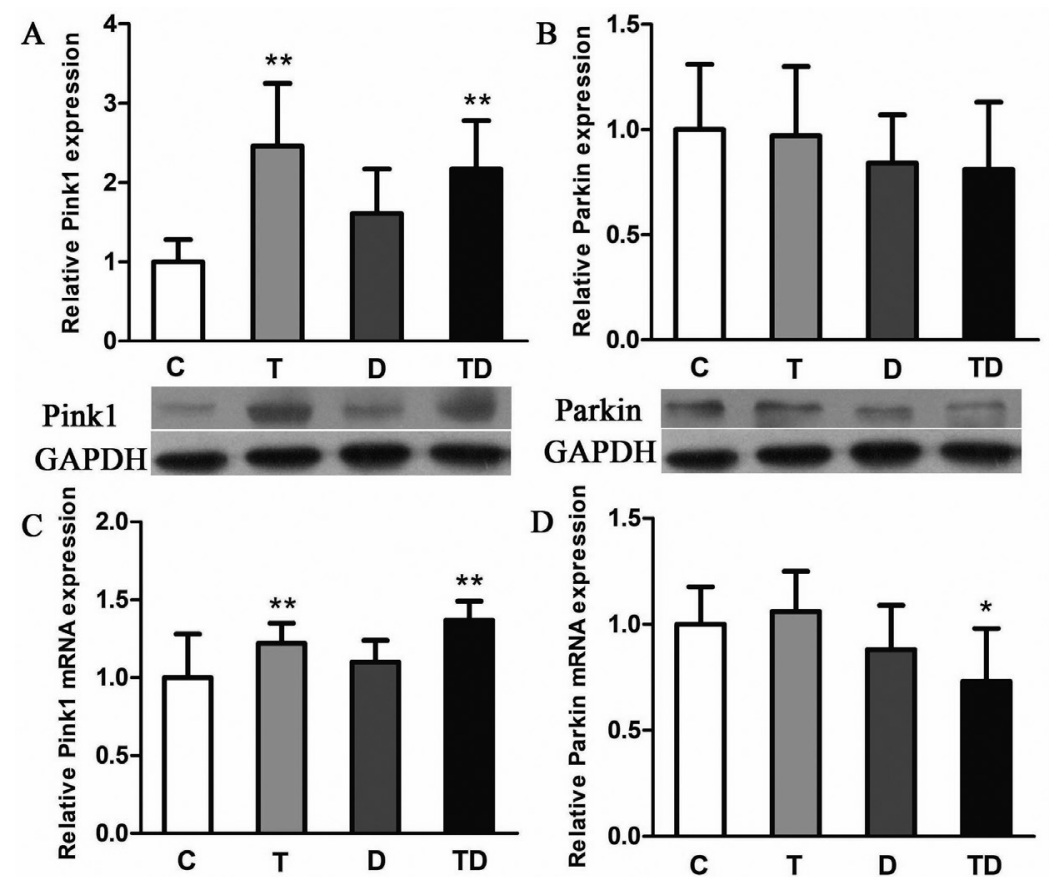

Figure 3. Changes of PINK1 and Parkin expressions after exercise training and dietary restriction. PINK levels were elevated (A), and Parkin did not change (B). Alteration in PINK mRNA was similar to the PINK1 protein $(\mathbf{C})$, and Parkin mRNA only significantly decreased in TD group (D). Representative blots of PINK1 and Parkin were placed below (A) and (B). Values are mean \pm SD. $n=8 /$ group, ${ }^{\star} p<0.05$ vs. C group, ${ }^{* *} p<$ 0.01 vs. C group. C, control group, T, exercise training group; $\mathrm{D}$, dietary restriction group; $\mathrm{TD}$, exercise training + dietary restriction group.

proliferating cells for their inability to dilute the damaged constituents through cell mitosis. Therefore, cardiomyocytes possess well-coordinated mechanisms to remove impaired mitochondria and replace them with new ones (Dorn 2016). Study indicates that, even at basal condition, mice have high level of mitophagy in the heart evaluated by Mito-Keima, suggesting the importance of mitophagy in the heart (Sun et al. 2015). Although it has been controversial for some inconsistencies on the effects of mitophagy stimulation in IR model, activation of mitophagy, especially in proper extent in physiological condition, seems to be protective for the heart (Andres et al. 2014; Yu et al. 2015). Our previous work suggested exercise training downregulates cardiac apoptosis and results in protective effects (Zhao and Ma 2016). It raises a question whether exercise training and dietary invention can lead to the same effects regarding PINK1/Parkin and receptor pathways involved in mitophagy.

In the current study, we focused on the morphologies of myofibrils and mitochondria, searching for autophagosomes randomly in visual fields. Consistent with our hypothesis, few autophagosomes can be found in $\mathrm{C}$ group but more emerge in T, D and TD groups, especially in TD group. Presence of these suspectable mitochondrial autophagosomes implied that exercise training or dietary restriction could provoke cardiac mitophagy, while it is exacerbated in TD group. There were no obvious abnormalities of myofibrils and mitochondria in $\mathrm{T}$ and $\mathrm{D}$ groups, suggesting that exercise training or dietary restriction did not lead to any pathological phenotype in ultrastructure. Moreover,
T group had more intact mitochondria and regular myofibrillar $\mathrm{Z}$ lines than other groups, suggesting that exercise training generates a favorable adaptation to metabolism. Unexpectedly, in contrast to the benefits induced by exercise, TD intervention resulted in substantial cardiomyocytes injuries evaluated by the dysfunctional mitochondria and abnormalities in $\mathrm{Z}$ lines. A study reported that young nonobese adults undergoing 3 months of modest exercise training with $12.5 \%$ reduction in energy intake have less mtDNA damage, more mtDNA content and antioxidant enzymes in skeletal muscle (Civitarese et al. 2007). Additionally, 40\% dietary reduction in our study may be insufficient to meet the minimal energy demand for mice, which combined with exercise could generate a prolonged intensive load, thereby resulting in ultrastructural dysfunction in cardiomyocytes. That may be the main reason for the failure to observe the benefits described as previous researches. It raises a question that whether a smaller range of energy reduction with exercise can bring about cardiac benefits, which need to be investigated in future.

PINK1/Parkin pathway is one of the most thoroughly studied molecular mechanisms of mitophagy. PINK1 functions as a stress sensor of mitochondria and is highly dependent on the mitochondrial membrane potential. PINK1 is imported into healthy mitochondria, where it is degraded at the inner mitochondrial membrane. When mitochondria are depolarized, PINK1 is stabilized and accumulates on OMM, where it phosphorylates several proteins, and these events recruit Parkin to the OMM, then initiating mitophagy (Jin et al. 2010; Matsuda et al. 2010). Mice deficient in PINK1 or 
Parkin show cardiac dysfunctions and pathological hypertrophy with gradual accumulation of impaired mitochondria, indicating the importance of PINK1/Parkin pathway in cardiomyocytes (Billia et al. 2011; Kubli et al. 2013). In the current study, T and TD groups showed increased levels of PINK1 significantly, but all interventions did not change Parkin expression. It seems that Parkin is more resistant to exercise and dietary interventions than PINK1. A growing body of evidence suggests that exercise can elevate autophagy (Rocchi and He 2017). However, most of these studies have been performed using autophagy biomarkers to evaluate autophagy but not mitophagy. A recent report showed that a bout of treadmill running in mice causes mitochondrial oxidative stress and enhances mitophagy in skeletal muscle evaluated by a fluorescent reporter gene (Laker et al. 2017). Acute exercise also can increase Parkin expression in skeletal muscle that is abolished after suppression of mitochondrial biogenesis (Vainshtein et al. 2015). It could be inferred that acute exercise rather than long-term training may influence Parkin apparently. It is complicated to explain the results that why the loads in T and TD group increased PINK1 but not Parkin. Previous studies indicated that deficiency in PINK1 leads to cardiac diseases rapidly, but inhibiting Parkin has slight influence on heart function especially in young mice (Billia et al. 2011; Kubli et al. 2013). It seems that, in physiological condition, PINK1 is more important than Parkin in regulating cardiac homeostasis. Exercise training alone or exercise training with limited nutrients in our experiment at least tagged dysfunctional mitochondria regarding the increase in PINK1. The tagged mitochondria may be removed through other unknown mechanisms that contribute to the enhanced mitophagy. PINK1 and Parkin did not change in $\mathrm{D}$ group implied other potential signaling may mediate the enhanced mitophagy.

A well-characterized mitophagy receptor pathway is Bnip3/Nix-mediated mechanism. Bnip3 and Nix are proapoptotic BH3-only family members and can interact directly with LC3 on the phagophore via their LIR, thereby tethering mitochondria to autophagosomes (Nah et al. 2017). Genetic ablation of Nix in mice leads to cardiac hypertrophy and decreased contractility with age, which is exaggerated in the condition of deficiencies in both Nix and Bnip3, suggesting that Bnip3 and Nix have overlapping functions in regulating mitochondrial turnover in cardiomyocytes (Dorn 2010). Our study did not find any upregulation in Bnip3 and Nix proteins levels in all groups. On the contrary, these proteins expressions decreased in $\mathrm{T}$ and TD groups significantly. The findings in our data suggest that exercise training decreased the Bnip3/Nix pathway and the frequent presence of suspectable mitochondrial autophagosomes may be attributed to other mitophagy machinery. Our findings differ from a study from Lira et al. (2013) in which 4 weeks of voluntary running resulted in increased expression of Bnip3 in parallel with enhanced mitochondrial biogenesis in plantaris. It appears that Bnip3 in myocardium and skeletal muscle exhibits distinctive responses upon exercise training. Of note, in the study from Lira et al. autophagy proteins of Bnip3 exhibited graded increases from vastus lateralis to plantaris and then to soleus muscles in basal condition,
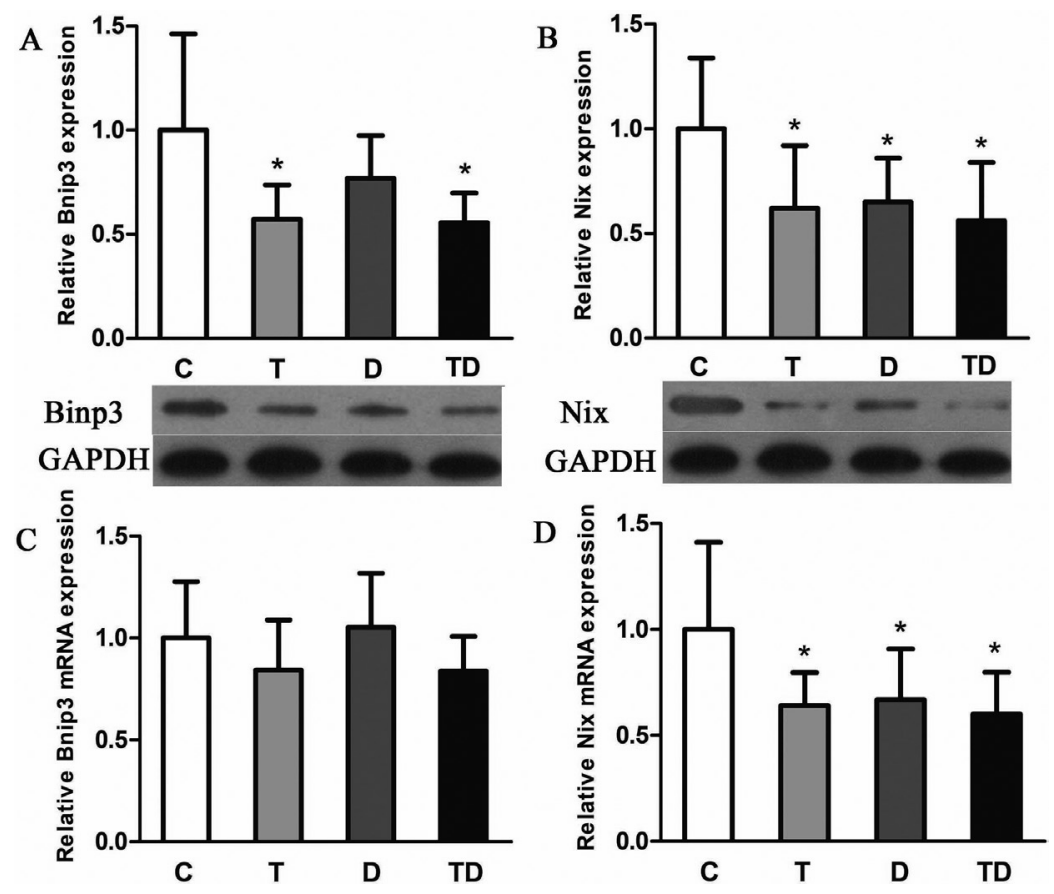

Figure 4. Changes of Bnip3 and Nix expressions after exercise training and dietary restriction. Bnip3 protein levels were reduced (A), and Nix exhibited the same change (B). Bnip3 mRNA expression changed but had no statistical significance (C). Nix mRNA expression decreased in concert with the change in protein (D). Representative blots of Bnip3 and Nix were placed below (A) and (B). Values are mean \pm SD. $n=8 /$ group, ${ }^{*} p<0.05 v s$. C group. For abbreviations, see Fig. 3. 

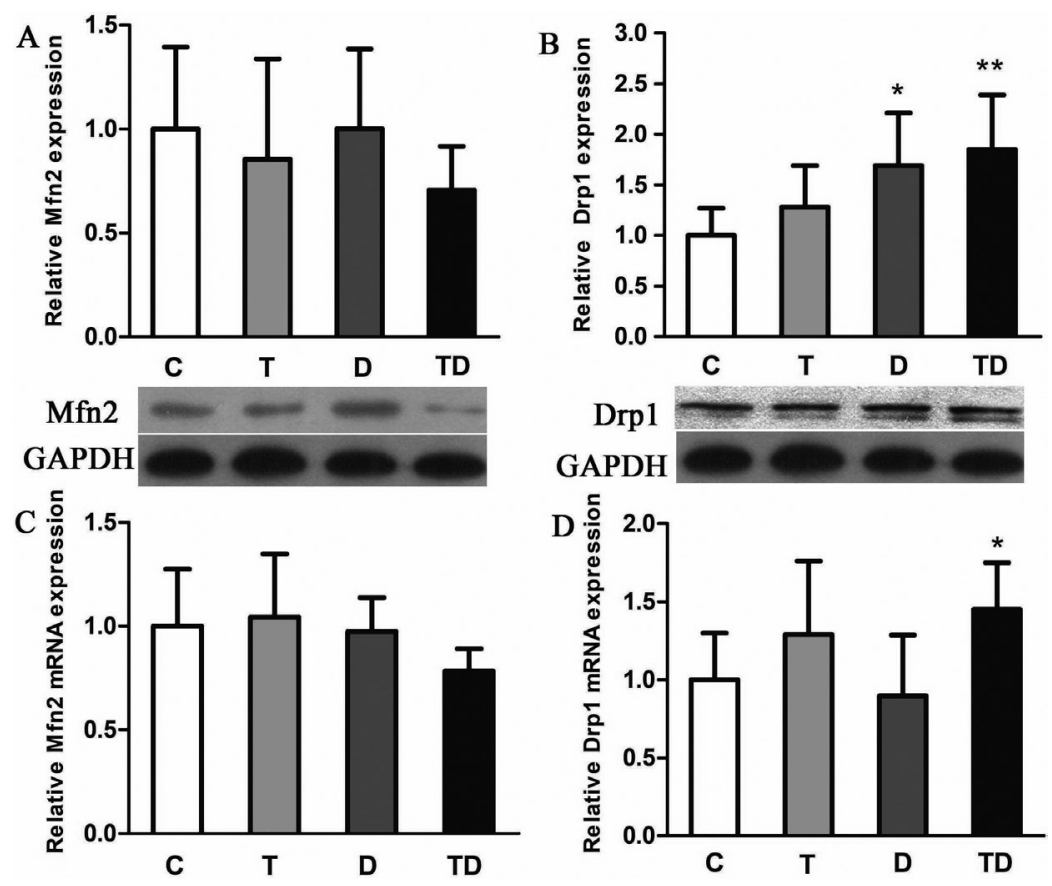

Figure 5. Changes of Mfn2 and Drp1 expressions after exercise training and dietary restriction. Mfn2 protein expression had decreased trends (A), whereas the levels of Drp1 protein increased in $\mathrm{D}$ and TD groups (B). Bnip3 mRNA expression was not affected in all groups (C). Drp1 mRNA expression increased apparently only in TD group (D). Representative blots of Mfn2 and Drp1 were placed below (A) and (B). Values are mean \pm SD. $n=8 /$ group, ${ }^{\star} p<0.05 v$ s. C group, ${ }^{\star *} p<0.01 v s$. $\mathrm{C}$ group. For abbreviations, see Fig. 3 . suggesting oxidative muscle rather than glycolytic muscle possessed higher mitophagy flux. Moreover, Bnip3 protein content is increased by exercise training in plantaris but not in soleus muscle. Together, it indicates Bnip3 is highly expressed in oxidative tissue and may not contribute to upregulation of mitophagy post exercise training. In our study, cardiomyocytes possess high level of oxidative capacity and the enhanced mitophagy may be independent of Bnip3/Nix. But cycling for $2 \mathrm{~h}$ at $70 \%$ maximal oxygen uptake intensity upregulated Bnip3 and Nix levels in human skeletal muscle (Schwalm et al. 2017). It seems that a bout of exercise rather than endurance exercise training upregulates Bnip3/Nix signaling. Dietary restriction had a trend to reduce the Bnip3 expression and reduced Nix expression significantly, suggesting the mitophagy response in D group may be mediated though other mechanisms. Changes of Bnip3 and Nix in TD group were similar to $\mathrm{T}$ group that indicated the mitophagy response in TD group is also independent of Bnip3/Nix pathway. Our study failed to detect the increases in Bnip3 and Nix in three protocols which may be relevant to the factor which specifically triggers the Bnip3/Nix pathway. Under hypoxic condition, elevation of mitophagy mediated by increased Bnip3 expression protects cells against ROS (Zhang et al. 2008). Both Bnip3 and Nix are involved in promoting mitophagy under hypoxia in cultured cells (Bellot et al. 2009). The intervention protocols in current study may not achieve the hypoxic demand that could be responsible for the unexpected result. Of note, mitophagy receptors should not be restricted to Bnip3 and Nix because new receptors are constantly identified such as Fun14 Domain containing 1 (FUNDC1), Bcl-2-like protein 13 (Bcl2-L-13) and Cardiolipin (Nah et al. 2017). We can not rule out the possibility that these underlying molecular mechanisms may be implicated in the mitophagy response in our study.

Mitochondrial fusion and fission also coordinate with mitophagy to maintain cardiac homeostasis. Mfn2 and Drp1 are highly expressed in mammalian heart, wherein their deficiencies inhibit cardiac mitophagy (Shirihai et al. 2015). Loss of Parkin receptor Mfn2 in the heart decreases PINK/Parkin-mediated mitophagy (Song et al. 2014). Drp1-mediated asymmetrical fission facilitates segregation of damaged mitochondrial components, leaving one severely damaged and one healthy mitochondrion. The damaged mitochondrion undergoes mitochondrial clearance (Shires and Gustafsson 2015). In the present study, Mfn2 did not change in all groups and Drp1 levels were increased in D and TD groups, implying that Mfn2 does not involve the mitophagy process in the three interventions. However, increased Drp1 may coordinates with other unknown signaling pathways to trigger mitophagy. Previous studies found decreased mitochondrial respiratory function and ATP in skeletal muscle was associated with decreased Mfn2 and increased Drp1 (Liu et al. 2014). Cardiac Mfn2 knockout mice also resulted in suppression of ATP synthesis (Mourier et al. 2015). Mitochondrial fusion facilitates ATP production but high excessive mitochondrial fission impairs oxidative phosphorylation (Mishra and Chan 2016). Exercise training in T group did not change Mfn2 and Drp1 expressions which meant 
energy metabolism in the heart operates in a normal condition, whereas increased Drp1 in D and TD groups may lead to excessive fission. The highest levels of Drp1 in TD group may result in serious energy metabolism dysfunction which could contribute to cardiomyocytes injuries.

Taken together, although the exercise training in our experiment did not alter Parkin expression, upregulation of PINK1 could promote the identification of the damaged mitochondria in the heart and facilitate the mitochondrial clearance. Intervention of $40 \%$ dietary reduction only increases Drp1 expression which was beneficial to orchestrate cardiac mitophagy. Exercise training and dietary restriction constituted a strenuous stress and led to cardiomyocytes injuries and enhanced mitophagy, which may be attributed to increases of PINK1, Drp1 and other molecular mechanisms. It is unknown why Bnip3 and Nix were downregulated in current study. Whether other molecular mechanisms cooperate to regulate mitophagy response induced by exercise and dietary restriction in the heart need to be further elucidated. Additionally, methods applied in present study only provided static information indicating what happened in molecular pathways of cardiomyocytes. But mitophagy is a highly dynamic process which includes formation of autophagosome, engulfment of mitochondria and lysosome-dependent degradation. It is necessary to examine the signaling in early and final stages of mitophagy in further studies. Our study also lacked parameters of cardiac functions evaluated by echocardiography pre- and post-interventions. Whether exercise training and dietary restriction result in beneficial or adverse effects in the cardiac function is unknown and the relation between mitophagy and cardiac function undergoing the intervention aforementioned also needs to be investigated.

Acknowledgments. This work was supported by the National Natural Science Foundation of China (31771316), and by the Research Project of Science and Technology Commission Foundation of Shanghai Municipality of China (15490503300).

Conflict of interest. The authors have no conflict of interest to declare.

\section{References}

Andres AM, Hernandez G, Lee P, Huang C, Ratliff EP, Sin J, Thornton CA, Damasco MV, Gottlieb RA (2014): Mitophagy is required for acute cardioprotection by simvastatin. Antioxid. Redox. Signal. 21, 1960-1973 https://doi.org/10.1089/ars.2013.5416

Baines CP (2010): The cardiac mitochondrion: nexus of stress. Annu. Rev. Physiol. 72, 61-80 https://doi.org/10.1146/annurev-physiol-021909-135929

Bellot G, Garcia-Medina R, Gounon P, Chiche J, Roux D, Pouyssegur J, Mazure NM (2009): Hypoxia-induced autophagy is medi- ated through hypoxia-inducible factor induction of BNIP3 and BNIP3L via their BH3 domains. Mol. Cell. Biol. 29, 2570-2581 https://doi.org/10.1128/MCB.00166-09

Billia F, Hauck L, Konecny F, Rao V, Shen J, Mak TW (2011): PTENinducible kinase 1 (PINK1)/Park6 is indispensable for normal heart function. Proc. Natl. Acad. Sci. USA 108, 9572-9577 https://doi.org/10.1073/pnas.1106291108

Chen Y, Dorn GW, 2nd (2013): PINK1-phosphorylated mitofusin 2 is a Parkin receptor for culling damaged mitochondria. Science 340, 471-475 https://doi.org/10.1126/science.1231031

Choi AM, Ryter SW, Levine B (2013): Autophagy in human health and disease. N. Engl. J. Med. 368, 651-662 https://doi.org/10.1056/NEJMra1205406

Civitarese AE, Carling S, Heilbronn LK, Hulver MH, Ukropcova B, Deutsch WA, Team CP (2007): Calorie restriction increases muscle mitochondrial biogenesis in healthy humans. PLoS. Med. 4, e76 https://doi.org/10.1371/journal.pmed.0040076

Dorn GW, 2nd (2010): Mitochondrial pruning by Nix and BNip3: an essential function for cardiac-expressed death factors. J. Cardiovasc. Transl. Res. 3, 374-383 https://doi.org/10.1007/s12265-010-9174-x

Dorn GW, 2nd (2016): Parkin-dependent mitophagy in the heart. J. Mol. Cell. Cardiol. 95, 42-49 https://doi.org/10.1016/j.yjmcc.2015.11.023

Hall AR, Burke N, Dongworth RK, Hausenloy DJ (2014): Mitochondrial fusion and fission proteins: novel therapeutic targets for combating cardiovascular disease. Br. J. Pharmacol. 171, 1890-1906 https://doi.org/10.1111/bph.12516

Ikeda Y, Shirakabe A, Maejima Y, Zhai P, Sciarretta S, Toli J, Sadoshima J (2015): Endogenous Drp1 mediates mitochondrial autophagy and protects the heart against energy stress. Circ. Res, 116, 264-278 https://doi.org/10.1161/CIRCRESAHA.116.303356

Jin SM, Lazarou M, Wang C, Kane LA, Narendra DP, Youle RJ (2010): Mitochondrial membrane potential regulates PINK1 import and proteolytic destabilization by PARL. J. Cell. Biol. 191, 933-942 https://doi.org/10.1083/jcb.201008084

Kubli DA, Zhang X, Lee Y, Hanna RA, Quinsay MN, Nguyen CK, Gustafsson AB (2013): Parkin protein deficiency exacerbates cardiac injury and reduces survival following myocardial infarction. J. Biol. Chem. 288, 915-926 https://doi.org/10.1074/jbc.M112.411363

Laker RC, Drake JC, Wilson RJ, Lira VA, Lewellen BM, Ryall KA, Yan Z (2017): Ampk phosphorylation of Ulk1 is required for targeting of mitochondria to lysosomes in exercise-induced mitophagy. Nat. Commun. 8, 548 https://doi.org/10.1038/s41467-017-00520-9

Lira VA, Okutsu M, Zhang M, Greene NP, Laker RC, Breen DS, Yan $\mathrm{Z}$ (2013): Autophagy is required for exercise training-induced skeletal muscle adaptation and improvement of physical performance. FASEB J. 27, 4184-4193 https://doi.org/10.1096/fi.13-228486

Liu R, Jin P, Yu L, Wang Y, Han L, Shi T, Li X (2014): Impaired mitochondrial dynamics and bioenergetics in diabetic skeletal muscle. PLoS One 9, e92810 
https://doi.org/10.1371/journal.pone.0092810

Matsuda N, Sato S, Shiba K, Okatsu K, Saisho K, Gautier CA, Tanaka K (2010): PINK1 stabilized by mitochondrial depolarization recruits Parkin to damaged mitochondria and activates latent Parkin for mitophagy. J. Cell. Biol. 189, 211-221 https://doi.org/10.1083/jcb.200910140

Mishra P, Chan DC (2016): Metabolic regulation of mitochondrial dynamics. J. Cell. Biol. 212, 379-387 https://doi.org/10.1083/jcb.201511036

Mourier A, Motori E, Brandt T, Lagouge M, Atanassov I, Galinier A, Larsson NG (2015): Mitofusin 2 is required to maintain mitochondrial coenzyme Q levels. J. Cell. Biol. 208, 429-442 https://doi.org/10.1083/jcb.201411100

Nah J, Miyamoto S, Sadoshima J (2017): Mitophagy as a protective mechanism against myocardial stress. Compr. Physiol. 7, 1407-1424 https://doi.org/10.1002/cphy.c170005

Peterson CM, Johannsen DL, Ravussin E (2012): Skeletal muscle mitochondria and aging: a review. J. Aging. Res. 2012, 194821 https://doi.org/10.1155/2012/194821

Psilander N, Frank P, Flockhart M, Sahlin K (2013): Exercise with low glycogen increases PGC-1alpha gene expression in human skeletal muscle. Eur. J. Appl. Physiol. 113, 951-963 https://doi.org/10.1007/s00421-012-2504-8

Quinsay MN, Thomas RL, Lee Y, Gustafsson AB (2010): Bnip3mediated mitochondrial autophagy is independent of the mitochondrial permeability transition pore. Autophagy 6, $855-862$

https://doi.org/10.4161/auto.6.7.13005

Rocchi A, He C (2017): Regulation of exercise-induced autophagy in skeletal muscle. Curr. Pathobiol. Rep. 5, 177-186 https://doi.org/10.1007/s40139-017-0135-9

Schwalm C, Deldicque L, Francaux M (2017): Lack of activation of mitophagy during endurance exercise in human. Med. Sci. Sports. Exerc. 49, 1552-1561 https://doi.org/10.1249/MSS.0000000000001256

Shires SE, Gustafsson AB (2015): Mitophagy and heart failure. J. Mol. Med. 93, 253-262 https://doi.org/10.1007/s00109-015-1254-6

Shirihai OS, Song M, Dorn GW, 2nd (2015): How mitochondrial dynamism orchestrates mitophagy. Circ. Res. 116, 1835-1849 https://doi.org/10.1161/CIRCRESAHA.116.306374

Song M, Chen Y, Gong G, Murphy E, Rabinovitch PS, Dorn GW, 2nd (2014): Super-suppression of mitochondrial reactive oxygen species signaling impairs compensatory autophagy in primary mitophagic cardiomyopathy. Circ. Res. 115, 348-353
https://doi.org/10.1161/CIRCRESAHA.115.304384

Sun N, Yun J, Liu J, Malide D, Liu C, Rovira II, Finkel T (2015): Measuring in vivo mitophagy. Mol. Cell. 60, 685-696 https://doi.org/10.1016/j.molcel.2015.10.009

Szafranski K, Mekhail K (2014): The fine line between lifespan extension and shortening in response to caloric restriction. Nucleus 5, 56-65 https://doi.org/10.4161/nucl.27929

Tolkovsky AM (2009): Mitophagy. Biochim. Biophys. Acta 1793, 1508-1515 https://doi.org/10.1016/j.bbamcr.2009.03.002

Vainshtein A, Tryon LD, Pauly M, Hood DA (2015): Role of PGC-1alpha during acute exercise-induced autophagy and mitophagy in skeletal muscle. Am. J. Physiol. Cell. Physiol. 308, C710-719 https://doi.org/10.1152/ajpcell.00380.2014

Watanabe T, Takemura G, Kanamori H, Goto K, Tsujimoto A, Okada H, Minatoguchi S (2014): Restriction of food intake prevents postinfarction heart failure by enhancing autophagy in the surviving cardiomyocytes. Am. J. Pathol. 184, 1384-1394 https://doi.org/10.1016/j.ajpath.2014.01.011

Youle RJ, Narendra DP (2011): Mechanisms of mitophagy. Nat. Rev. Mol. Cell. Biol. 12, 9-14 https://doi.org/10.1038/nrm3028

Youle RJ, van der Bliek AM (2012): Mitochondrial fission, fusion, and stress. Science 337, 1062-1065 https://doi.org/10.1126/science.1219855

Yu P, Zhang J, Yu S, Luo Z, Hua F, Yuan L, Xu G (2015): Protective effect of sevoflurane postconditioning against cardiac ischemia/ reperfusion injury via ameliorating mitochondrial impairment, oxidative stress and rescuing autophagic clearance. PLoS One 10, e0134666 https://doi.org/10.1371/journal.pone.0134666

Zhang H, Bosch-Marce M, Shimoda LA, Tan YS, Baek JH, Wesley JB, Semenza GL (2008): Mitochondrial autophagy is an HIF1 -dependent adaptive metabolic response to hypoxia. J. Biol. Chem. 283, 10892-10903 https://doi.org/10.1074/jbc.M800102200

Zhao Y, Ma Z (2016): Swimming training affects apoptosis-related microRNAs and reduces cardiac apoptosis in mice. Gen. Physiol. Biophys. 35, 443-450 https://doi.org/10.4149/gpb_2016012

Received: April 23, 2018

Final version accepted: June 15, 2018

First published online: November 15, 2018 